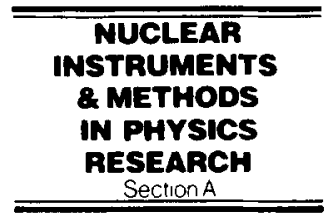

\title{
Impact of variations in physical parameters on glow curves for planchet heating of TL dosimeters is
}

\author{
E. Samei ${ }^{\text {a,* }}$, K.J. Kearfott ${ }^{\text {a }}$, C-K.C. Wang ${ }^{b}$, S. Han ${ }^{b}$ \\ ${ }^{a}$ Department of Nuclear Engineering, College of Engineering, The University of Michigan, Ann Arbor, MI 48109, USA \\ ${ }^{\mathrm{b}}$ Health Physics Program, School of Mechanical Engineering, Georgia Institute of Technology, Atlanta, GA 30332, USA
}

\begin{abstract}
This study consists of a theoretical analysis of the directional planchet heating of Thermoluminescent Dosimeters (TLD) with an emphasis on influence of radiation field type, TL material properties, and heating scheme parameters on the resulting glow curve. Computer software is developed to simulate the thermal conduction and TL production processes in a planchet-heated TLD chip. The results of the simulation are benchmarked to previous experimental findings for a LiF TLD and excellent agreement is obtained. The system thermophysical parameters and initial depth-dose distribution in the TLD are varied and the position of the main glow peak and integral glow are examined. A demonstration is given of how a set of thermophysical parameters may provide information about the depth-dose distribution in the TLD and how variation in the values of these parameters may limit the reconstruction of this depth-dose information.
\end{abstract}

\section{Introduction}

Contact heating, or so-called planchet heating, has been widely used as a heating modality for thermoluminescent dosimeters (TLDs). Conventionally, the amount of time-integrated thermoluminescence (TL) light during the heating period is utilized to estimate the total dose absorbed in the dosimeter. A differential heating approach has recently been proposed for individual monitoring in beta and betagamma mixed radiation fields with the underlying concept of production of a high thermal gradient in the TL element to yield a glow curve structure that can be unfolded to reconstruct the initial distribution of trapped carriers or depth-dose distribution in the dosimeter [1,2]. Because unfolding algorithms used in this approach are sensitive to the shape of the glow curve, in particular the time of onset of the peak, any non-dose-dependent variations of the shape may greatly influence the results. Other investigators have previously studied the effects of variation in thermal resistance at the contact area between the TLD chip and the planchet on the resulting glow curve $[3,4]$. The scope of this paper is to show quantitatively how other thermophysical parameters such as chip thickness, bulk TL absorption in the chip, thermal conduction coefficient, and heating rate may influence the resulting glow curve and

\footnotetext{
The work is supported by U.S. Department of Energy contract No. DE-FG02-92ER 75703.

* Corresponding author.
}

consequently limit the reconstruction of depth-dose information in a differential heating prospective.

\section{Methodology}

A computer code is written to simulate the heat transfer and TL production processes in a planchet-heated TLD chip. To represent the thermal conduction process, a onedimensional model is implemented which assumes free convection with the surrounding gas at the upper surface and imperfect thermal contact at the lower surface of the TLD chip [3]. The time-dependent temperature profiles computer by this model are then combined with the first order Randall-Wilkins TL model, which elucidates for detrapping of electrons, neglects retrapping, and assumes pseudo-steady state conditions [5]. The time-dependent intensity of TL signal that reaches the TL light detector and the total Iuminescence from TLD are calculated by integration of positional TL over TLD volume and heating time, accounting for attenuation and reflection of TL in the chip.

The computer simulation requires that the thermophysical properties of the system depth-dose distribution in the TLD chip be entered upon execution. A LiF TLD is simulated which its physical properties are estimated from those for pure, sintered, or PTFE crystals [4,6,7]. The depth-dose distributions produced in beta fields are modeled assuming an exponential absorption behavior in the TLD chip [8]. The depth-dose distributions generated by 
photon irradiation are considered uniform since the interaction probability between photons and TL material is smaller than the one associated with low-penetrating beta radiation by about two order of magnitude and therefore, in a relatively thin cross-sectional profile of a TLD chip, the depth-dose distribution is practically uniform [9]. The distributions generated by ${ }^{137} \mathrm{Cs}$ gamma and ${ }^{90} \mathrm{Sr} /{ }^{90} \mathrm{Y}$ beta radiation fields are also implemented using EGS4, a Monte Carlo radiation transport code [10]. Since the interest is to observe the influence of the form of the depth-dose distribution on the glow characteristics rather than the total radiation dose absorbed in the dosimeter, distributions are normalized to their maximum values.

The source code is run on a UNIX workstation and output data are processed on a personal computer using commercially available statistics and graphics software packages. The positional distribution of temperature, TL signal production in the TLD chip, and spatially integrated glow curves are obtained for a number of cases. In each run, the thermophysical parameters of the system are varied within a certain range corresponding to expected experimental variations. Specifically, the effects of chip thickness, heating rate, thermal conduction, contact roughness, TL bulk attenuation, and initial depth-dose distribution in the chip are studied. As a measure of change in the shape of the glow curve, the position of the main glow curve peak and the area under the glow curve (integral glow) are examined. The uncertainties associated with peak position and integral glow are determined by introducing random variations to each of the physical parameters within their certain expected fluctuations and performing a statistical analysis.

\section{Results and discussion}

Temperature profiles generated for a planchet-heated LiF TLD were similar to the ones illustrated elsewhere $[3,4]$. Those profiles indicate that the production of a thermal gradient across the TLD chip approaches a constant value with time. As heat flows from the bottom to the top, different layers of the TL chip experience different temperature histories and reach their maximum TL emission at different times. This temporally variant thermal gradient confirms the underlying concept of the differential heating approach: information about the depth at which positional TL emission occurs may be generated by deconvolution of a folded glow curve. The simulated glow curve was benchmarked to the previous findings [11] and excellent agreement was obtained.

The effects of changing the heating rate on the position of the main peak of the glow curve is illustrated in Fig. 1. Increasing the heating rate shifts the peaks to the left and the amount of the shift slowly decreases for higher heating rates. This illustrates that a higher heating rate is more desirable in a differential heating approach; it produces a

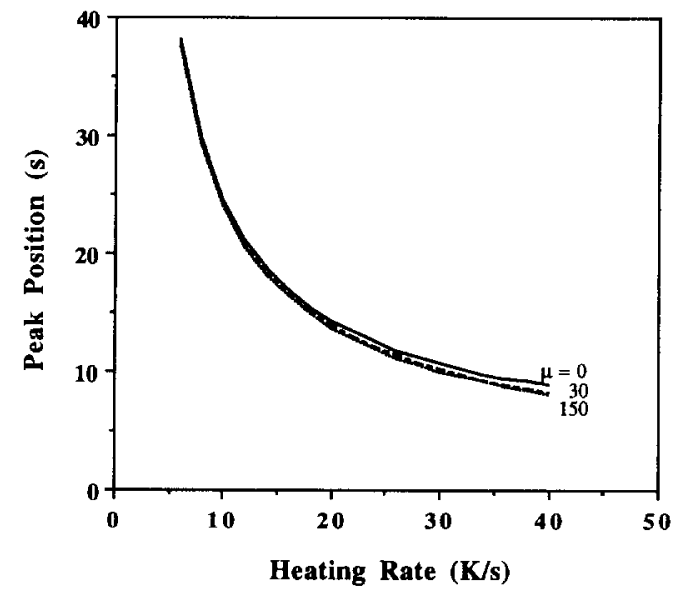

Fig. 1. Variation of peak position with heating rate calculated for different linear absorption coefficient of beta rays in $\mathrm{cm}^{-1}$.

more depth-dose-dependent peak position while the peak position is less sensitive to the variations in the heating rate. This behavior is expected since at higher heating rates, the heat propagation is primarily limited by the heat conduction property of TL material itself. Alternatively, the amount of integral glow is independent of the heating rate for any given depth-dose distribution. The flatness of response is due to the fact that the amount of integral glow is directly related to the total number of trapped carriers in the chip and is independent of their time-dependent luminescence.

The effect of variation in the thermal contact resistance between the lower surface of the TLD chip and the heating element on the peak position is shown in Fig. 2. Increasing the contact roughness linearly shifts the main glow peak toward later times regardless of the depth-dose distribu-

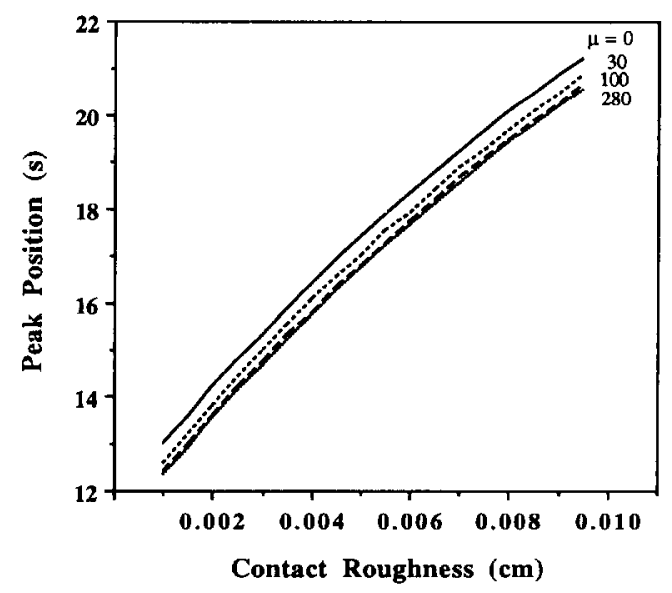

Fig. 2. Variation of peak position with thermal contact roughness (thermal resistance) calculated for different linear absorption coefficient of beta rays $\mathrm{in} \mathrm{cm}^{-1}$. 


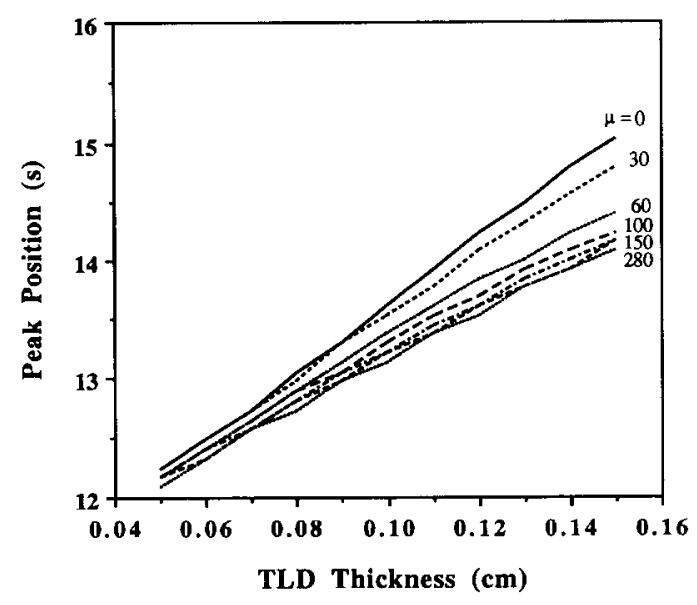

Fig. 3. Variation of peak position with TLD thickness calculated for different linear absorption coefficient of beta rays in $\mathrm{cm}^{-1}$.

tion in the chip. This demonstrates that the contact resistance merely acts as a barrier to slow down the heat conduction process from the heating element to the TLD chip. Again, due to the same reason stated above, the amount of integral glow was found to be independent of the variation in the thermal resistance.

Fig. 3 illustrates the effect of variation in the chip thickness on the peak position. As the chip gets thicker and thicker, the peak position shifts to the right and this shift is less rapid and closer to linear if the positional dose is located closer to the heating element. As the positional dose is more uniformly distributed in the chip, peak position-thickness relationship gets farther from linear. This behavior can be described by two means: First, increasing the thickness of the chip causes a larger thermal gradient across the dosimeter. This, on the one hand, enhances the contribution of depth-dose distribution to the position of the peak. On the other hand, since different layers of the chip experience different temperatures at different times, they do not reach to the point of their maximum luminescence at the same time and the main peak position of the resulting glow curve is a summation of these "off-phase" peaks generated by different layers of the TL chip. Secondly, varying the thickness affects the resulting peak position by changing the attenuation of TL light in the chip. As the TL signal is attenuated in the TLD, the layers of the dosimeter that are farther from the light detector contribute less to the glow curve than the ones which are closer. Fig. 3 clearly shows that glow curve resulting from a thicker dosimeter is more sensitive to the depth-dose distribution in the chip and is more appropriate to be used in a differential heating approach than the ones resulting from a thinner dosimeter.

The same two reasons given above explain the variations in the amount of integral glow with element's thickness which are illustrated in Fig. 4. In the case of uniform

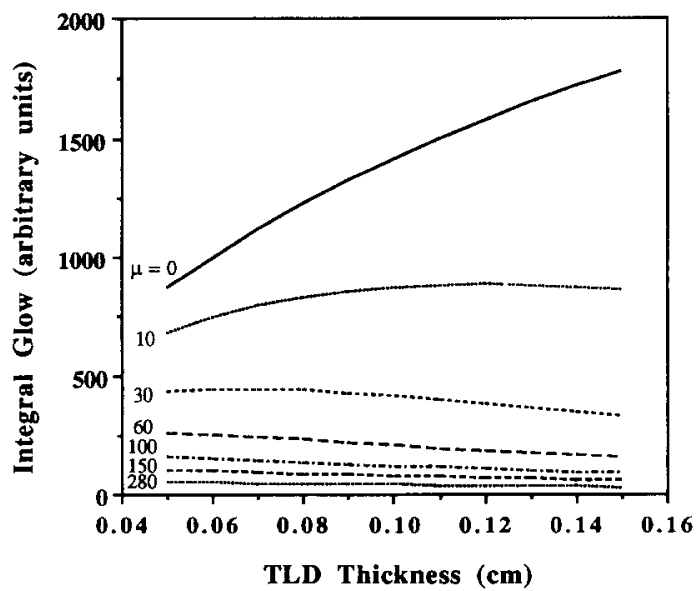

Fig. 4. Variation of integral glow with TLD thıckness calculated for different linear absorption coefficient of beta rays in $\mathrm{cm}^{-1}$.

depth-dose distribution, the total cumulative dose in the chip linearly increases with thickness. However, the amount of integral glow does not increase quite linearly due to attenuation of TL signal in the chip. When the positional dose is more concentrated toward the heating element, the amount of integral glow, as thickness increases, becomes more and more affected solely by the TL attenuation.

Fig. 5 illustrates the effects of the thermal conduction coefficient of the TL material on the peak position of the resulting glow curve. The thermal conduction coefficient has smaller impact on the position of the main peak in the glow curve when the positional dose is concentrated closer to the heating element. At lower values of thermal conduction, the peak position becomes very sensitive to the depth-dose distribution. However, in the low-conduction region, the peak position is also quite sensitive to varia-

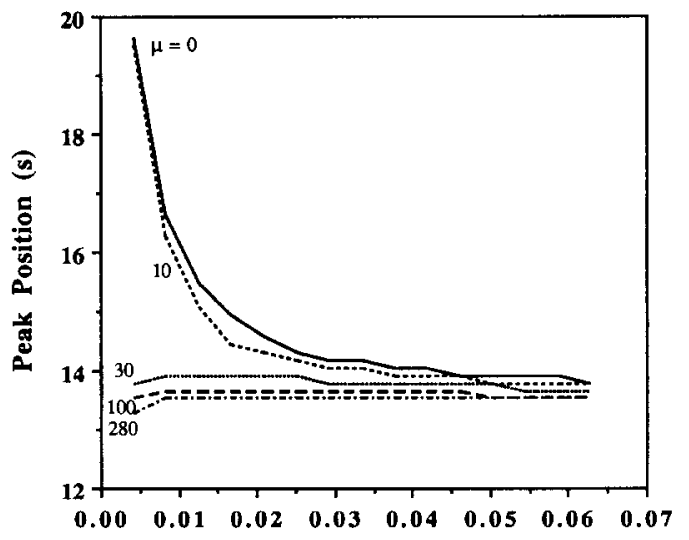

TLD Thermal Conduction Coefficient $\left(\mathrm{J} / \mathrm{s}-\mathrm{cm}^{2}-\mathrm{K}\right)$

Fig. 5. Var1ation of peak position with thermal conduction of TLD calculated for different linear absorption coefficient of beta rays in $\mathrm{cm}^{-1}$. 


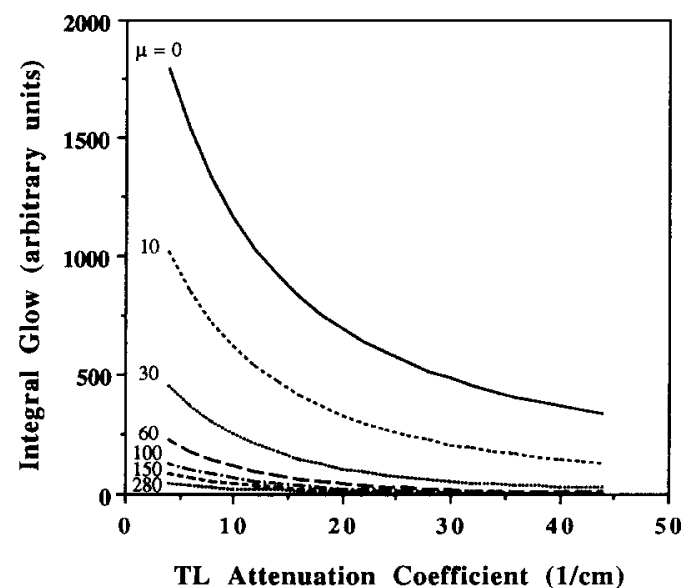

Fig. 6. Variation of integral glow with TL attenuation coefficient calculated for different linear absorption coefficient of beta rays in $\mathrm{cm}^{-1}$

tions in the thermal conduction coefficient. The amount of integral glow, on the other hand, is independent of the amount of thermal conduction coefficient. The flatness of integral glow response may again be described by independence of integral glow from time-related processes in the chip during the heating stage.

Varying the bulk attenuation of TL in the chip, it was found that the peak position is independent of TL attenuation coefficient. This behavior is expected since the TL bulk attenuation is purely a non-time-related process. A high attenuation coefficient, however, may limit the sensitivity of the systems based on deconvolution of the glow curve; a higher attenuation produces a lower detected TL signal and therefore a higher statistical uncertainty of the results. Fig. 6 illustrates the effects of variation in the attenuation coefficient of TL material on the amount of integral glow. Integral glow is exponentially reduced as the TL attenuation coefficient increases.

The exclusive variation of depth-dose distribution in the chip resulted the relationships shown in Fig. 7. Both peak position and integral glow are strongly dependent on the form of the depth-dose distribution. However, integral

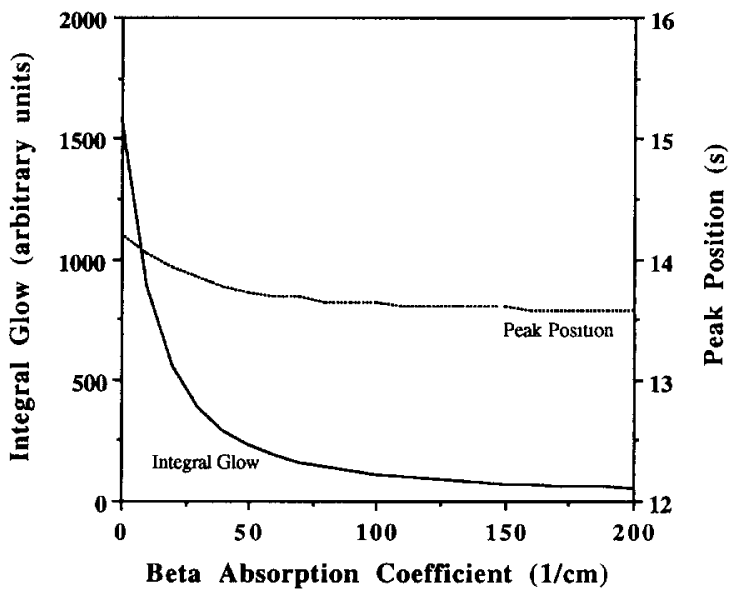

Fig. 7. Dependence of TL peak position and integral glow on the linear absorption coefficient of beta rays.

glow demonstrates a stronger dependence. This is due to the fact that the amount of integral glow mainly reflects the total absorbed dose in the dosimeter which is quite sensitive to the exponential fallout of depth-dose distribution, recalling the normalization of distributions to their optimum values.

All of the quantities discussed above together have an overall effect on the peak position and the amount of integral glow. Table 1 compares the contribution of variation in each of these quantities to the overall effect in a typical experimental setup. Each parameters has been varied by a certain amount corresponding to expected experimental variations. The data show that for a given variation in each of the quantities, with the exception of TL attenuation, the amount of integral glow is more stable than the peak position. The uncertainty evaluation, considering the accumulative influence of variation in each of the physical parameters, resulted relative uncertainties of $2.28 \%$ for peak position and $1.54 \%$ for integral glow. With a pronounced variation of the radiation field from ${ }^{137} \mathrm{Cs}$ gamma rays to ${ }^{90} \mathrm{Sr} /{ }^{90} \mathrm{Y}$ beta rays, only $1.29 \%$ shift in the glow curve was observed which is quite comparable with the ones caused by fluctuations of other parameters. This

Table 1

Summary of impact of experimental variations in thermophysical parameters on the resulting glow curve Each parameter has been varied independently. The set parameters were as indicated in the table without variations

\begin{tabular}{llllc}
\hline Parameter & Variation & Units & $\begin{array}{l}\text { Maximum shift of } \\
\text { main glow peak }\end{array}$ & $\begin{array}{l}\text { Maximum change } \\
\text { in integral glow }\end{array}$ \\
\hline Heating rate & $15.0 \pm 10 \%$ & $\mathrm{~K} \mathrm{~s}^{-1}$ & $1.22 \%$ & $0.5 \%$ \\
Contact roughness & $0.002 \pm 50 \%$ & $\mathrm{~cm}$ & $5.94 \%$ & $0.14 \%$ \\
Thickness & $0.14 \pm 0.005$ & $\mathrm{~cm}$ & $5.94 \%$ & $1.76 \%$ \\
TL attenuation & $5.6 \pm 10 \%$ & $\mathrm{~cm}^{-1}$ & $0.0 \%$ & $0.62 \%$ \\
Heat conduction & $2.51 \times 10^{3} \pm 10 \%$ & $\mathrm{~J} \mathrm{~s}^{-1} \mathrm{~cm}^{-2} \mathrm{~K}^{-1}$ & $8.38 \%$ & $0.10 \%$ \\
Dose distribution & ${ }^{137} \mathrm{Cs}$ gammas & - & $1.29 \%$ & depends on the \\
& ${ }^{90} \mathrm{Sr} /{ }^{90} \mathrm{Y}$ betas & & & field strength \\
\hline
\end{tabular}


demonstrates an obvious drawback of directional planchet heating in the differential heating approach for the extraction of depth-dose information from the shape of the glow curve.

\section{Conclusion}

Thermophysical parameters such as heating rate, contact region resistance, thermal conduction, convection coefficient, and dosimeter dimensions have significant impacts on the position of the resulting glow curve in planchet-heated TLDs. The depth-dose distribution in the TLD chip also influences the shape of the glow curve. In this regard, selection of a thick TLD along with low temperature conductivity and high heating rate may produce large thermal gradient in the TLD chip which results a glow curve shape capable of providing information about the depth-dose distribution. However, experimental fluctuation of thermophysical parameters can vary the shape of the glow curve such that these variations may overlap the small variations due to the influence of depth-dose distribution. This limits the application of planchet heating in methods based on deconvolution of the glow curve. The amount of integral glow, on the other hand, shows a higher stability comparing to the peak position in regard to statistical uncertainty in thermophysical parameters while it is quite sensitive to the depth-dose distribution in the chip. This demonstrates superiority of a conceptual system for beta and mixed field dosimetry that works based on the amount of total integral glow. These results also confirm that an assumption of a uniform depth-dose distribution in the chip in a conventional approach may lead to a high error in estimation of dose in the fields that low penetrating radiation exists.

\section{References}

[1] P. Sahre, Nucl. Instr. and Meth. 217 (1983) 525.

[2] P. Sahre, Radiat. Protect. Dosim. 39 (1991) 139.

[3] V.I. Gotlib, L.N. Kantorovich, V.L. Grebenshicov, V.R. Bichev and E.A. Nemiro, J. Phys. D 17 (1984) 2097.

[4] V.I. Gotlib, V.L. Grebenshicov, L.N. Kantorovich and P. Sahre, Radiat. Protect. Dosim. 22 (1988) 13.

[5] J.T. Randall and M.H.F. Wilkins, Proc. Conf. Royal Soc. London A 184 (1945) 366.

[6] S.W.S. McKeever, Nuc. Instr. and Meth. 175 (1980) 19.

[7] M. Grupen and K. Kearfott, J. Appl. Phys. 64 (1988) 1044.

[8] B.R. Paliwal and P.R. Almond, Health Phys. 31 (1976) 151.

[9] F.H. Attix, Introduction to Radiological Physics and Radiation Dosimetry (Wiley, New York, NY, 1986).

[10] W.R. Nelson, H. Hirayama and D.W.O. Rogers, The EGS4 Code System, Stanford Lin. Accel. Report SLAC-265 (1985).

[11] Harshaw-Bicron Radiation Measurement Products, 6801 Cochran Road, Solon, $\mathrm{OH} 44139$, USA. 